\title{
Nine degree of freedom motion estimation for wrist PPG heart rate measurements
}

DOI:

10.1109/EMBC.2019.8857100

\section{Document Version}

Accepted author manuscript

Link to publication record in Manchester Research Explorer

\section{Citation for published version (APA):}

Vazquez Galvez, A., \& Casson, A. (2019). Nine degree of freedom motion estimation for wrist PPG heart rate measurements. In 2019 41st Annual International Conference of the IEEE Engineering in Medicine and Biology Society (EMBC) (IEEE Engineering in Medicine and Biology Society. Conference Proceedings). https://doi.org/10.1109/EMBC.2019.8857100

Published in:

2019 41st Annual International Conference of the IEEE Engineering in Medicine and Biology Society (EMBC)

\section{Citing this paper}

Please note that where the full-text provided on Manchester Research Explorer is the Author Accepted Manuscript or Proof version this may differ from the final Published version. If citing, it is advised that you check and use the publisher's definitive version.

\section{General rights}

Copyright and moral rights for the publications made accessible in the Research Explorer are retained by the authors and/or other copyright owners and it is a condition of accessing publications that users recognise and abide by the legal requirements associated with these rights.

\section{Takedown policy}

If you believe that this document breaches copyright please refer to the University of Manchester's Takedown Procedures [http://man.ac.uk/04Y6Bo] or contact uml.scholarlycommunications@manchester.ac.uk providing relevant details, so we can investigate your claim.

\section{OPEN ACCESS}




\title{
Nine degree of freedom motion estimation for wrist PPG heart rate measurements
}

\author{
Arturo Vazquez Galvez and Alexander J. Casson, Senior Member, IEEE
}

\begin{abstract}
In recent years there has been substantial interest in wearable devices that measure heart rate via photoplethysmography (PPG) sensors placed at the wrist. This is challenging as the wrist PPG signal is severely corrupted by artefacts during motion, and although a number of algorithms are now available commercially and academically there is still a need for improved performance, especially when examining physical activities other than running. To date, algorithms for motion artefact removal from the PPG have focused on the use of a co-located accelerometer to record the motion. In this work, we introduce co-located accelerometer, gyroscope and magnetometer sensors to allow three, six and nine degrees of freedom estimates of the motion present. Assessed during a bike riding task the results show that the heart rate estimation is improved by up 0.57 beats per minute by using the additional information from these new sensors.
\end{abstract}

\section{INTRODUCTION}

The interest in wearable sensors for monitoring vital signs has increased significantly in recent years [1]. The focus of these devices is to have a constant monitoring of parameters such as heart rate and blood pressure [2], as well as many applications in longitudinal observations of elderly users and improving athletes performance [3]. The use of portable biometrics on a daily lifestyle is increasing, with many applications that encourage the users to exercise and keep track of their achievements [4]. Heart rate is usually measured via photoplethysmography (PPG) which shines a light into the skin and measures the amount of light reflected, which changes with blood flow. This approach has been used for a long time for stationary subjects, but PPG signals are severely corrupted by motion artefacts when the user moves around [5] which until recently has prohibited their use in a wearable manner. To solve this issue, many different signal processing methodologies have been implemented in order to filter out the motion artefact in the signal (e.g. [5], [6], [7]). These build on the 2015 IEEE signal processing cup [5] where PPG data from 23 subjects during running exercise was released publicly, and on this data set several algorithms report error rates in a range of $1-2$ beats per minute (bpm), or $2-3 \%$ if the range of the mean value is considered. However, the practical error rates reported when devices such as the Apple Watch, Samsung Gear, or Fitbit are used in the real-world are somewhat higher than this. Taking the mean value range of each output for resting, walking and running, the

*This work was supported by Mexico's National Council of Science and Technology (CONACYT).

The authors are with the School of Electrical and Electronic Engineering, The University of Manchester, Manchester, UK. Email: arturo.vazquezgalvez@manchester.ac.uk, alex.casson@manchester.ac.uk performance of commercial devices such Apple Watch falls under the $2 \%$ percent range for resting, $10 \%$ for walking stage of exercise and $7 \%$ for jogging exercises [8].

In this paper, we investigate the above in two ways. Firstly by incorporating additional co-located sensors into the PPG recording we analyze PPG signals where accelerometery, gyroscope, and magnetometer traces have been recorded at the same time and location on the wrist. For the first time this allows 3 degree of freedom (using only the accelerometery), 6 degree of freedom (using the accelerometery and gyroscope) and 9 degree of freedom (using all 3 additional sensors) estimates of the motion to be calculated and used for motion artefact removal. We demonstrate how this use of sensor fusion can lead to an improvement in the heart rate estimation provided. This builds upon our previous work [9] where we only compared the accelerometer and gyroscope signals and did not fuse the two together to give an improved overall estimation of motion. Secondly, we verify our approach using data recording during bike riding, to demonstrate the performance heart rate estimation across a wider set of physical activities and databases compared to previous works.

The remainder of this paper is organized as follows. Section II gives our methods which are based upon the use of an adaptive filter with the motion corrupted PPG signal and an estimation of the motion present as the inputs. It then gives the methods for fusing the three sets of motion sensor data together to estimate the overall motion present at the wrist, and a summary of the data used. Results are given in Section III with these discussed and conclusions drawn in Section IV.

\section{METHODS}

We make use of an adaptive filter previously reported for removing motion artefacts from PPG signals [10] as our starting point as it has two inputs: the motion corrupted PPG signal, and an estimation of the motion present, the "noise" input. We can then readily switch this noise input between our three different estimations of motion present, based upon the use of the different co-located sensors. Fig. 1 shows an overview of all the stages in the algorithm. At the start, the input signals (raw PPG) and "noise" data are put through a band-pass filtered (Butterworth) and resampled to $256 \mathrm{~Hz}$. In the next stage, the data is processed by normalized mean square (NMLS) adaptive filters, one filter for each "noise" input corresponding to motion in the $\mathrm{x}, \mathrm{y}$, and $\mathrm{z}$-axes respectively. These inputs are themselves bandpass filtered, with a third order Butterworth filter $(0.4-4 \mathrm{~Hz})$ prior to processing. 


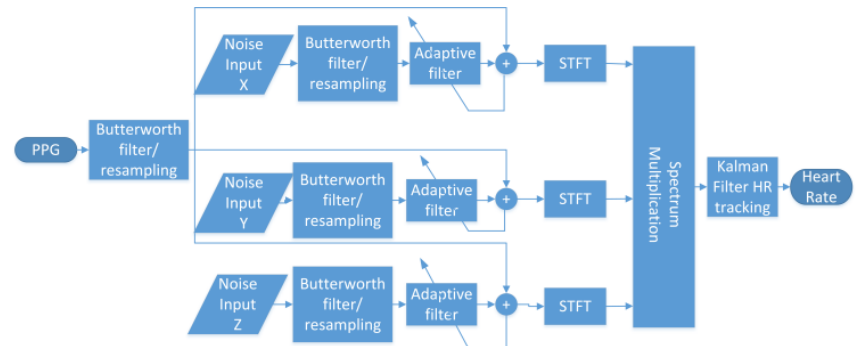

Fig. 1. Heart rate tracking algorithm overview based upon that from [11] with an improved Kalman filter at the output for spectral peak tracking.

Heart rate estimation is performed in the frequency domain with the cleaned PPG traces after adaptive filtering are passed to a Short Time Fourier Transforms (STFT) with settings a normalized least mean square adaptive filter order set to 9 , its step size set to 0.1 and the number of points is set to 4096. In the end, a single combined spectral signal is obtained by a multiplying each STFT output, so that the dominant spectral peak due to heart rate and present in all of the traces is maintained while residual noise components tend to average out. The heart rate estimate is obtained by tracking the dominant power frequency in the combined spectrogram using a Kalman filter with the following state model.

$$
\begin{gathered}
\boldsymbol{K} \boldsymbol{G}_{\boldsymbol{t}}=\frac{\boldsymbol{E}_{\boldsymbol{s t}}}{\boldsymbol{E}_{\boldsymbol{s t}}+\boldsymbol{E}_{\boldsymbol{m}}} \\
\boldsymbol{E}_{\boldsymbol{s t}}=\left[\mathbf{1}-\boldsymbol{K} \boldsymbol{G}_{\boldsymbol{t}-\mathbf{1}}\right]\left(\boldsymbol{E}_{\boldsymbol{s t}-\mathbf{1}}\right) \\
H_{t}=H_{t-1}+K G\left[M_{t}-H_{t-1}\right]
\end{gathered}
$$

where $K G$ is the Kalman gain (at sample $t$ or $t-1$ ), $E_{s t}$ is the error estimate at time sample $t, E_{m}$ is the error measurement, $M$ is the measured peak frequency and $H_{t}$ is the heart rate estimate. Our algorithm is implemented offline in MATLAB, and analyses $8 \mathrm{~s}$ windows of data, with $6 \mathrm{~s}$ of overlap between subsequent windows (which these settings mirror those used for many papers analyzing the IEEE signal processing cup data).

Accelerometers measure the rate of change of velocity, while gyroscopes measure the rate of change of angular velocity, and magnetometers measure the movement of the sensor through the Earth's magnetic field (and other interfering fields from electrical equipment and similar). These multiple sensors can be used individually, as in our previous work [9], or can be fused to generate one overall estimation of motion. In this paper, we investigate three different methods of combining motion measurements.

1) Three degrees of freedom (3DoF): Accelerometer only. This analysis uses only the accelerometer data as the "noise" input to the adaptive filter. It is thus equivalent to that reported previously in [10] and the many PPG motion artefact removal papers which make use of the IEEE cup data which only have accelerometer information available [5], [6] [13]. As pre-processing, the acceleration due to gravity is subtracted based on which axis is being affected at any specific moment. Based on the work presented in [14], to determine which axis is "facing down", there are six probable positions where axis $\mathrm{x}, \mathrm{y}$ or $\mathrm{z}$ can be directly affected by gravity in a positive or negative manner. As an example if the maximum acceleration occurs on the $\mathrm{Y}$-axis, is negative, and lies within the range $-\mathrm{g} \pm 0.5 \mathrm{~g}$, we denote the corresponding orientation of the sensor as position 1 .

2) Six degrees of freedom (6DoF): Accelerometer and gyroscope. With the addition of the gyroscope data, an additional analysis can be performed since the gyroscope is not affected by static gravity acceleration and instead measures angular velocity [15]. To obtain the angular position a cumulative trapezoidal numerical integration was performed to each axis $(\mathrm{x}, \mathrm{y}, \mathrm{z})$ of the gyroscope where the limits were established by the time axis of the entire signal depending on the duration of the test. With these angular position values, the orientation of the sensor with respect to the ground can be determined by following a procedure from [16], this takes the raw data recorded in the frame of reference ( $\mathrm{x}, \mathrm{y}, \mathrm{z}$ directions) which moves as the sensor does, and projects them onto a fixed set of axes relative to the ground which allows gravity to be easily subtracted from the $\mathrm{z}$-direction. For each axis in the fixed space, the required rotation matrixes for the sensor's $x, y$ and z-axes are represented in Fig. 2, where the axis that is facing "towards" the screen is being projected.

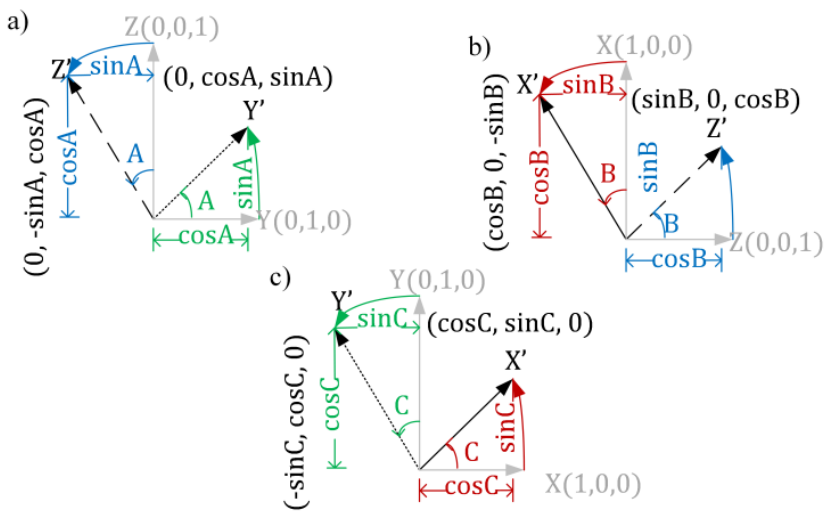

Fig. 2. Rotation of a) x-axis (solid line, red highlights), b) y-axis (dotted line, green highlights) and c) z-axis (dashed line, blue highlights) [17].

If we take the $\mathrm{x}$-axis as an example, the initial values of $\mathrm{y}$ and $\mathrm{z}$ are $(0,1,0)$ and $(0,0,1)$. If $\mathrm{x}$-axis rotates, $\mathrm{y}^{\prime}$ becomes $(0, \cos A, \sin A)$ and $z^{\prime}$ becomes $(0,-\sin A, \cos A)$. The new axes are inserted as column components of a $3 \times 3$ rotation matrix. Each axis is represented in equation 4, 5 and 6. These can be combined into one matrix that will vary depending on their order (starting from $R_{x} R_{y} R_{z}$ to $R_{z} R_{y} R_{x}$ ), which is defined by the accelerometer axes, where the most dominant position is determined for each activity. 


$$
\begin{aligned}
R_{x} & =\left(\begin{array}{ccc}
1 & 0 & 0 \\
0 & \cos A & -\sin A \\
0 & \sin A & \cos A
\end{array}\right) \\
R_{y} & =\left(\begin{array}{ccc}
\cos B & 0 & \sin B \\
0 & 1 & 0 \\
-\sin B & 0 & \cos B
\end{array}\right) \\
R_{z} & =\left(\begin{array}{ccc}
\cos C & -\sin C & 0 \\
\sin C & \cos C & 0 \\
0 & 0 & 1
\end{array}\right)
\end{aligned}
$$

The gravity force effect is added by obtaining the product of the rotation matrix and the gravity factor, as shown in equation 7 , where $S_{z}$ is taken from the bottom row of the resulting matrix and $\mathrm{g}$ is gravity $\left(9.8 \mathrm{~m} / \mathrm{s}^{2}\right)$ [18].

$$
S_{g}=g \times S_{z}
$$

The motion of the sensor can be obtained by removing the gravity factor from the acceleration data with equation 8 , where $S_{a}$ is the accelerometer data.

$$
\tilde{S}_{a}=S_{a}-S_{g}
$$

3) Nine degrees of freedom (9DoF): accelerometer, gyroscope and magnetometer. The use of all three sensors allows for the absolute position and orientation of the wearable device to be calculated using quaternions [19]. The quaternion can be defined as a way to represent rotation, with a three-dimensional vector and a scalar number, which as the name implies, consists of four floating-point numbers to make up the vector and scalar components of the quaternion [20]. Here we make use of the implementation provided in [19] which has a MATLAB script available for download. The basic product is summarised in (9) and Fig. 3 , which describe an arbitrary rotation from one frame (that of the sensor) to another (a fixed frame which does not change with movement). With the implementation of quaternions in the data analysis, it is now possible to find the absolute sensor position at each point in time.

$$
{ }_{B}^{A} \hat{q}=\left[q_{1} q_{2} q_{3} q_{4}\right]=\left[\cos \frac{\theta}{2}-r_{x} \sin \frac{\theta}{2}-r_{y} \sin \frac{\theta}{2}-r_{z} \sin \frac{\theta}{2}\right]
$$

Fig 3. Orientation of frame $B$ is achieved by a rotation, from alignment with frame $A$, of angle $\theta$ around the vector $\boldsymbol{A}_{\hat{r}}$ [19].

The data that is analyzed was previously reported in [21]. Eight records of wrist PPG recorded during bike riding are available, with simultaneous accelerometer, gyroscope and magnetometer recordings. Subjects cycled on a bike for periods of approximately 5 minutes, with different levels of resistance present. Wrist signals were collected using a Shimmer 3 [22] wearable device. A reference chest electrocardiogram (ECG) was also recorded using a
camNtech actiwave [23] to allow a gold-standard comparison of heart rate. All signals recorded at $256 \mathrm{~Hz}$ and the Shimmer and ECG trace manually synchronized.

\section{RESULTS AND DISCUSSION}

A comparison between the merged STFT outputs for the different degrees of freedom configurations is shown in Fig. 4 (corresponding to record 6). For this record, visually all are broadly similar, with the underlying heart rate trajectory being tracked. In the six degrees of freedom case in particular (Fig. 4b) it can be seen that less low-frequency noise is present, and therefore has the most accurate estimate of heart rate. The differences in performance are quantified for all records in Table 1, using the Average Absolute Error (AAE) as defined in [13]. On average, the improvement is best for the six degrees of freedom case, with a 0.57 beats per minute improvement. The nine degree of freedom case sees a mean improvement of 0.31 beats per minute. Significant variations are seen between different records, for example, in record 1 the error actually is increased when using more degrees of freedom compared to the accelerometer only, while in record 2 a substantial decrease from around $6.3 \mathrm{bpm}$ error to around $2.3 \mathrm{bpm}$ is obtained by using the additional sensors. In record 5 it is the nine degree of freedom method which obtains the best

\begin{tabular}{|c|c|c|c|}
\hline Record & Sensors & Resistance level & $\mathrm{AAE}(\mathrm{bpm})$ \\
\hline \multirow{3}{*}{1} & $3 \mathrm{DoF}$ & \multirow{3}{*}{ Low resistance } & 2.50 \\
\hline & $6 \mathrm{DoF}$ & & 2.97 \\
\hline & 9DoF & & 2.65 \\
\hline \multirow{3}{*}{2} & 3DoF & \multirow{3}{*}{ High resistance } & 6.33 \\
\hline & 6DoF & & 2.25 \\
\hline & 9DoF & & 2.34 \\
\hline \multirow{3}{*}{3} & 3DoF & \multirow{3}{*}{ Low resistance } & 3.19 \\
\hline & $6 \mathrm{DoF}$ & & 2.27 \\
\hline & 9DoF & & 3.29 \\
\hline \multirow{3}{*}{4} & 3DoF & \multirow{3}{*}{ High resistance } & 2.76 \\
\hline & $6 \mathrm{DoF}$ & & 1.97 \\
\hline & 9DoF & & 3.00 \\
\hline \multirow{3}{*}{5} & 3DoF & \multirow{3}{*}{ Low resistance } & 3.82 \\
\hline & $6 \mathrm{DoF}$ & & 4.25 \\
\hline & 9DoF & & 3.03 \\
\hline \multirow{3}{*}{6} & 3DoF & \multirow{3}{*}{ High resistance } & 2.64 \\
\hline & $6 \mathrm{DoF}$ & & 2.08 \\
\hline & 9DoF & & 3.30 \\
\hline \multirow{3}{*}{7} & 3DoF & \multirow{3}{*}{ Low resistance } & 6.72 \\
\hline & $6 \mathrm{DoF}$ & & 5.26 \\
\hline & 9DoF & & 6.48 \\
\hline \multirow{3}{*}{8} & 3DoF & \multirow{3}{*}{ Low resistance } & 5.65 \\
\hline & $6 \mathrm{DoF}$ & & 7.98 \\
\hline & 9DoF & & 7.01 \\
\hline \multirow{3}{*}{ Mean } & 3DoF & \multirow{3}{*}{ Overall } & 4.20 \\
\hline & $6 \mathrm{DoF}$ & & 3.63 \\
\hline & 9DoF & & 3.89 \\
\hline
\end{tabular}
performance. This shows that the best motion estimation is different for different records, and indeed different methods may be better at different points in time within the same record.

Table 1. Degrees of freedom comparison for PPG data collected during bicycle riding at a low and high resistance level.

Based on the data shown in Table 1, the nine degrees of freedom could improve the overall performance when compared to the three degrees of freedom analysis but did 
not improve the six degrees of freedom analysis, which can be attributed to the quality of the signal that comes from the sensor.

Having additional sensors turned on will increase the power consumption of the wearable device, and in future work, this may provide the opportunity to trade-off between power consumption and accuracy with an automatic switching between different estimations of motion.

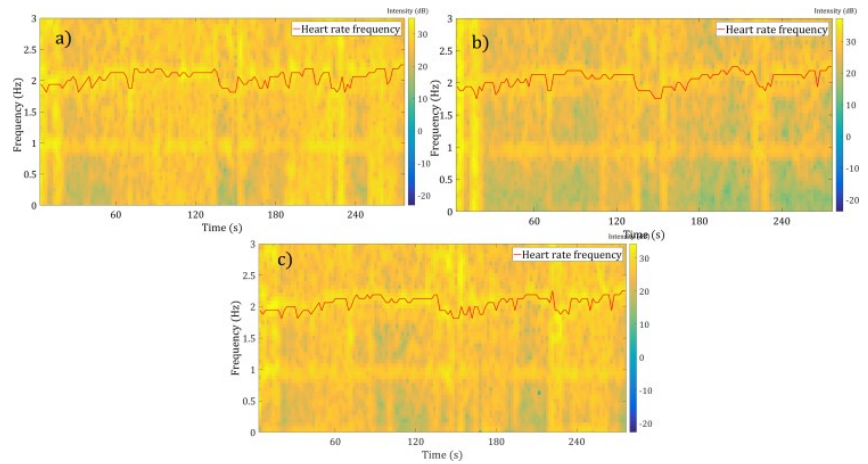

Fig. 4: Spectrograms for: a) three, b) six and c) nine degrees of freedom analysis of PPG data. Red line shows the tracked heart rate estimate.

\section{CONCLUSIONS}

Overall, the integration of accelerometer, gyroscope and magnetometer data into the motion artefact removal process has provided better results when compared to just using accelerometer data, however, applying a 9DoF method did not improve the results over using a $6 \mathrm{DoF}$ approach, this can be credited to the sensitivity of the magnetometer sensor. As it can be seen in Fig. 4, using magnetometer can produce similar outputs and even help at times, however, on average it leads to a worse hr estimate, consumes more sensor power, due to the power consumption of the actual sensors and its more complex fusing algorithms.

Today few wearable PPG devices contain all three types of sensors and this may provide an opportunity for improving the heart rate estimation, overall considering the performance in accuracy and potential battery power consumption, with the six degrees of freedom method providing the most accurate average estimates for bike riding exercise, it is the recommended method in most situations. For future work, it would be beneficial to analyze an approach where the different configurations based on the degrees of freedom can be swapped when it is most convenient.

\section{REFERENCES}

[1] M. Chan, D. Estève, J.-Y. Fourniols, C. Escriba, and E. Campo, "Smart wearable systems: Current status and future challenges," Artif Intell Med, vol. 56, no. 3, pp. 137-156, 2012.

[2] S. Ghosh, A. Banerjee, N. Ray, P. W. Wood, P. Boulanger, and R. Padwal, "Using accelerometric and gyroscopic data to improve blood pressure prediction from pulse transit time using recurrent neural network," 2018 IEEE Int. Conf. Acoust. Speech Signal Process., pp. 935-939, 2018.

[3] L. Yanfen and H. Pu, "Smart Sportswear," 2011 Int. Conf. Futur. Comput. Sci. Educ., pp. 135-138, 2011.
[4] I. M. Albaina, T. Visser, C. a P. G. van der Mast, and M. H Vastenburg, "Flowie: A persuasive virtual coach to motivate elderly individuals to walk," Int. ICST Conf. Pervasive Comput. Technol. Healthc., pp. 1-7, 2009.

[5] Z. Zhang, Z. Pi, and B. Liu, "TROIKA: A general framework for heart rate monitoring using wrist-type photoplethysmographic signals during intensive physical exercise," IEEE Trans. Biomed. Eng., vol. 62 , no. 2, pp. 522-531, 2015.

[6] E. Khan, F. Al Hossain, S. Zia Uddin, S. Kaisar Alam, and M. Kamrul Hasan, "A robust heart rate monitoring scheme using photoplethysmographic signals corrupted by intense motion artifacts," IEEE Trans. Biomed. Eng., vol. 63, no. 3, pp. 550-562, 2016.

[7] X. Zhang and X. Zhang, "Stretchablity and piezoelectricity of irradiation cross-linked polypropylene films with space charges," 2013 Annu. Rep. Conf. Electr. Insul. Dielectr. Phenomena, CEIDP, pp. 214-217, 2013.

[8] Z. Ge, P. W. C. Prasad, N. Costadopoulos, A. Alsadoon, a. K. Singh, and A. Elchouemi, "Evaluating the accuracy of wearable heart rate monitors," 2016 Int. Conf. Adv. Comput. Commun. Autom. (Fall), ICACCA 2016, pp. 1-6, 2016.

[9] Alexander, J. Casson, A. Vazquez, and D. Jarchi, "Gyroscope vs. accelerometer measurements of motion from wrist PPG during physical exercise," ICT Express, vol. 2, pp. 175-179, 2016.

[10] T. Schack, C. Sledz, M. Muma, and A. M. Zoubir, "A new method for heart rate monitoring during physical exercise using photoplethysmographic signals," 2015 23rd Eur. Signal Process. Conf. EUSIPCO 2015, pp. 2666-2670, 2015.

[11] D. Jarchi and A. J. Casson, "Estimation of heart rate from foot worn photoplethysmography sensors during fast bike exercise," Proc. Annu. Int. Conf. IEEE Eng. Med. Biol. Soc. EMBS, vol. 2016-Octob, pp. 3155-3158, 2016.

[12] M. Van Biezen, "iLectureOnline," 2016. [Online]. Available: http://www.ilectureonline.com/.

[13] A. Temko, "PPG-based heart rate estimation using Wiener filter, phase vocoder and viterbi decoding," 017 IEEE Int. Conf. Acoust. Speech Signal Process., pp. 1013-1017, 2017.

[14] D. Biswas, D. Corda, G. Baldus, A. Cranny, K. Maharatna, J. Achner, J. Klemke, M. Jöbges, and S. Ortmann, "Recognition of elementary arm movements using orientation of a tri-axial accelerometer located near the wrist.," Physiol. Meas., vol. 35, no. 9, pp. 1751-68, 2014.

[15] M. Mashhadi, E. Asadi, M. Eskandari, S. Kiani, and F. Marvasti, "Heart rate tracking using wrist-type photoplethysmographic (PPG) signals during physical exercise with simultaneous accelerometry," IEEE Signal Process. Lett., vol. 23, no. 2, 2016.

[16] Z. Shilin, T. Ke, Z. Xinyu, L. Zhiqiang, and L. Bin, "MICROST: A mixed approach for heart rate monitoring during intensive physical exercise using wrist-type PPG signals," 2015 37th Annu. Int. Conf. IEEE Eng. Med. Biol. Soc., pp. 2347-2350, 2015.

[17] S. H. Ahn, "OpenGL angles to axes," 2015. [Online]. Available: http://www.songho.ca/opengl/gl_anglestoaxes.html.

[18] J. K. Lee, E. J. Park, and S. N. Robinovitch, "Estimation of attitude and external acceleration using inertial sensor measurement during various dynamic conditions," IEEE Trans. Instrum. Meas., vol. 61, no. 8, pp. 2262-2273, 2012.

[19] S. Madgwick, "An efficient orientation filter for inertial and inertial/magnetic sensor arrays," Tech. Rep. x-io , Univ. Bristol, vol. 25, pp. 113-118, 2010.

[20] S. Sanei, C. C. Took, and S. Enshaeifar, "Quaternion adaptive line enhancer based on singular spectrum analysis," 2018 IEEE Int. Conf. Acoust. Speech Signal Process., pp. 2876-2880, 2018.

[21] D. Jarchi and A. Casson, "Description of a database containing wrist PPG signals recorded during physical exercise with both accelerometer and gyroscope measures of motion," Data, vol. 2, no. 1, pp. 1-11, 2017.

[22] ShimmerSensing, "IMU User guide," 2014. [Online]. Available: www.shimmersensing.com.

[23] camNtech, "Cam n Tech cardio features," 2011. [Online]. Available: www.camntech.com. 